\title{
Magnetoencephalogram Blind Source Separation and Component Selection Procedure to Improve the Diagnosis of Alzheimer's Disease Patients
}

\author{
Javier Escudero*, Student Member, IEEE, Roberto Hornero, Member, IEEE, Daniel Abásolo, \\ Member, IEEE, Alberto Fernández, and Jesús Poza, Student Member, IEEE
}

\begin{abstract}
The aim of this study was to improve the diagnosis of Alzheimer's disease (AD) patients applying a blind source separation (BSS) and component selection procedure to their magnetoencephalogram (MEG) recordings. MEGs from 18 AD patients and 18 control subjects were decomposed with the algorithm for multiple unknown signals extraction. MEG channels and components were characterized by their mean frequency, spectral entropy, approximate entropy, and LempelZiv complexity. Using Student's $t$-test, the components which accounted for the most significant differences between groups were selected. Then, these relevant components were used to partially reconstruct the MEG channels. By means of a linear discriminant analysis, we found that the BSS-preprocessed MEGs classified the subjects with an accuracy of $80.6 \%$, whereas $72.2 \%$ accuracy was obtained without the BSS and component selection procedure.
\end{abstract}

\section{INTRODUCTION}

$S^{n}$ INCE their appearance, blind source separation (BSS) techniques [1] have been applied to the analysis of electroencephalogram (EEG) and magnetoencephalogram (MEG) signals [2]. Making several assumptions about the data structure, BSS methods can estimate the underlying components of a multichannel recording blindly (i.e., the real mixing process and inner components are unknown) [1], [2]. These algorithms can isolate dissimilar types of physiological activity into different components. Hence, BSS has been extensively applied to remove artifacts from EEG and MEG data [2]-[4]. However, other approaches are possible when applying BSS to EEGs or MEGs. Given the inherent complexity of brain signals, some components of the EEG or MEG might be more sensitive to particular brain states than others [5], [6]. Thus, the most relevant components may be selected to partially reconstruct the signals so that a better differentiation between specific brain states might be achieved [5]. Thus, BSS might be a valuable preprocessing technique to help in the diagnosis of important

Manuscript received March 21, 2007. This work was supported in part by the grant project VA108A06 from "Consejería de Educación de Castilla y León" and by the "Ministerio de Educación y Ciencia" and FEDER grant MTM 2005-08519-C02-01. Asterisk indicates corresponding author.

J. Escudero*, R. Hornero, D. Abásolo, and J. Poza are with the Biomedical Engineering Group, E.T.S.I. Telecomunicación, University of Valladolid, Camino del Cementerio s/n, 47011-Valladolid, Spain (phone: +34 983 423000, ext. 5589; fax: +34 983 423667; e-mail: javier.escudero@ieee.org).

A. Fernández is with the Centro de Magnetoencefalografía Dr. PérezModrego, Universidad Complutense de Madrid, Spain. mental impairments like Alzheimer's disease (AD).

$\mathrm{AD}$ is the most frequent degenerative neurological disease among elderly people in western countries [7], [8]. It causes a progressive and irreparable impairment of mental functions [7]. Moreover, $\mathrm{AD}$ can only be definitely diagnosed by necropsy [7]. Thus, the usefulness of EEG and MEG signals in $\mathrm{AD}$ diagnosis has been extensively researched in the last decades [9]. EEG and MEG record the neural currents with high temporal resolution [10]. Moreover, both recordings can be analyzed using several methods to gain a better understanding of $\mathrm{AD}$. For instance, straightforward spectral features such as mean frequency $\left(f_{\text {mean }}\right)$ [11], [12] or spectral entropy $(S p E n)$ [12] have quantified the abnormalities in the spectra of AD patients' EEG and MEG recordings [9]. Moreover, nonlinear statistics, like Lempel-Ziv complexity $(L Z C)$ [13] or approximate entropy $(A p E n)$ [14], can provide additional information about the brain dynamics in $\mathrm{AD}$ [9].

In this preliminary study, we applied a BSS algorithm to MEG recordings from control subjects and AD patients. We wanted to test whether a BSS and a component selection procedure might improve the subject classification when straightforward features like $f_{\text {mean }}, S p E n, L Z C$ or $A p E n$ were used to characterize MEG recordings.

\section{MATERIAL AND METHODS}

\section{A. Subjects and MEG recording}

The MEG recordings were acquired from 36 subjects. Eighteen patients ( 7 men and 11 women) who fulfilled the criteria of probable AD were recruited from the "Asociación de Enfermos de Alzheimer" (AFAL). Their average age was $72.78 \pm 8.61$ years - mean \pm standard deviation $(\mathrm{SD})-$. All $\mathrm{AD}$ patients were diagnosed following the guidelines provided by the National Institute of Neurological and Communicative Disorders and Stroke - Alzheimer's Disease and Related Disorders Association (NINCDS-ADRDA) [15]. The mini-mental state examination (MMSE) [16] score was $17.61 \pm 3.97$ (mean $\pm \mathrm{SD}$ ). No patient was receiving medication that could affect the MEG.

MEG was also recorded from 18 elderly control subjects (7 men and 11 women; $72.06 \pm 5.51$ years, mean $\pm \mathrm{SD}$ ) without past or present mental diseases. The control subjects' MMSE score was $29.11 \pm 0.90$ (mean \pm SD).

All AD patients' caregivers and control subjects gave their informed consent to take part in the current research, 
which was approved by the local ethics committee.

The MEG recording was performed in a magnetically shielded room with a 148-channel whole-head magnetometer (MAGNES $2500 \mathrm{WH}$, 4D Neuroimaging). Five minutes of MEG were acquired from each of the subjects while they were lying on a patient bed with eyes closed in a relaxed state. The sampling frequency was $678.19 \mathrm{~Hz}$. Nevertheless, the recordings were down-sampled to $169.549 \mathrm{~Hz}$ to reduce their length. Afterward, the data were digitally filtered between $1.5 \mathrm{~Hz}$ and $40 \mathrm{~Hz}$. Finally, MEG epochs of 20 seconds (3390 data points) that were simultaneously artifact-free at all channels were selected for further analysis.

\section{B. Blind source separation (BSS) algorithm}

BSS methods estimate de set of $n$ unknown components, $\mathbf{s}(t)=\left[s_{1}(t), \ldots, s_{n}(t)\right]^{\mathrm{T}}$, which were linearly mixed by the full rank $n \times n$ matrix $\mathbf{A}$ to form $n$ temporally and spatially correlated recordings, $\mathbf{x}(t)=\left[x_{1}(t), \ldots, x_{n}(t)\right]^{\mathrm{T}}[1]$, [2]. Thus, $\mathbf{x}(t)$ represents the MEG signals:

$$
\mathbf{x}(t)=\mathbf{A s}(t) .
$$

Making several assumptions [1] which have been previously validated for EEG and MEG data [2], some BSS algorithms use the temporal dependences of $\mathbf{x}(t)$ to compute a demixing matrix, $\mathbf{W}$. Using $\mathbf{W}$, the estimated components, $\mathbf{y}(t)=\left[y_{1}(t), \ldots, y_{n}(t)\right]^{\mathrm{T}}$, are recovered by [2]-[5]:

$$
\mathbf{y}(t)=\mathbf{W} \mathbf{x}(t) .
$$

Some BSS components may be more affected by AD than others [5]. Thus, a subset of the most sensitive components $\mathbf{y}_{p}(t)$ - may be back projected to the channels using the inverse of $\mathbf{W}, \mathbf{W}^{-1}$, to compute a partial reconstruction of the MEGs $-\mathbf{x}_{p}(t)$ :

$$
\mathbf{x}_{p}(t)=\mathbf{W}^{-1} \mathbf{y}_{p}(t) .
$$

$\mathbf{x}_{p}(t)$ may have enhanced specific features of AD. Thus, a better classification of $\mathrm{AD}$ patients versus control subjects may be achieved [5]. However, a fixed order is needed to compare components from different epochs and subjects [5], [6]. Hence, we employed the algorithm for multiple unknown signals extraction (AMUSE) [1], [17], which orders the components by linear predictability [5]. AMUSE decorrelates the input signals at two times delays [2], [17] (usually, at lags $\tau=0$ and $\tau=1$ samples [1], [4], [5]). Similarly to these studies, we decorrelated the MEG epochs at time delays $\tau=0$ and $\tau=1$ samples.

\section{Feature extraction}

MEG channels and AMUSE components were characterized by two spectral features $\left(f_{\text {mean }}\right.$ and $\left.S p E n\right)$ and two nonlinear statistics ( $A p E n$ and $L Z C$ ).

1) Mean frequency $\left(f_{\text {mean }}\right)$

$f_{\text {mean }}$ summarizes the spectral content of a signal. It has already been used to study brain signals in AD [9], [11], [12]. Firstly, the power spectral density $(P S D)$ of the signal is computed as the Fourier transform of its autocorrelation function [12]. Then, the $P S D$ is normalized $\left(P S D_{n}\right)$ and the $f_{\text {mean }}$ is estimated as the average frequency of the $P S D_{n}$ with a logarithmic transformation to get a distribution closer to the Gaussian one:

$$
f_{\text {mean }}=\exp \left[\sum_{f=1.5 \mathrm{~Hz}}^{40 \mathrm{~Hz}} P S D_{n}(f) \log (f)\right] .
$$

\section{2) Spectral entropy (SpEn)}

$S p E n$ has been applied to AD patients' EEG and MEG data to measure the flatness of the spectrum [12]. Broad and flat spectra produce high $S p E n$ values, whereas narrower $P S D$ s have lower $S p E n$ values [18]. It is estimated applying the Shannon's entropy to the $P S D_{n}[18]$ :

$$
S p E n=\frac{-1}{\log (N)} \sum_{f=1.5 \mathrm{~Hz}}^{40 \mathrm{~Hz}} P S D_{n}(f) \log \left[P S D_{n}(f)\right],
$$

where $N$ is the number of frequency bins and $\log (N)$ normalizes the $S p E n$ to a range from 0 to 1 .

3) Lempel-Ziv complexity (LZC)

Recently, $L Z C$ has been widely used to estimate the complexity in the Kolmogorov's sense of various biomedical signals [19], [20]. LZC is a non-parametric statistic that measures the number of distinct substrings and their rate of recurrence along the signal. It assigns larger values to more complex data [19]. Moreover, LZC can provide useful information about the MEGs of $\mathrm{AD}$ patients [13]. The algorithm to compute the $L Z C$ can be found in [13] or [20].

4) Approximate entropy (ApEn)

In 1991, Pincus [21] introduced the ApEn as a family of statistics to quantify the regularity of time series by evaluating the appearance of repetitive patterns [22]. ApEn has been used on biological data, such as AD patients' EEG recordings, providing potentially useful information [14], [22]. ApEn has two parameters: a run length $m$ and a tolerance window $r$ [21]. In this study, ApEn was estimated with the commonly used parameter values of $m=1$ and $r=$ 0.25 times the $\mathrm{SD}$ of the original time series [22]. The implementation of the ApEn is detailed in [21] or [22].

\section{Statistical analysis}

Student's $t$-test was used to decide whether there were significant differences between both subject groups in the $f_{\text {mean }}, S p E n, L Z C$, and $A p E n$ values of the AMUSE components. Normality and homoscedasticity were verified with Shapiro-Wilk and Levene's test, respectively.

A linear discriminant analysis (LDA) with a leave-one-out cross-validation procedure was used to compute the increase in the classification accuracy of the subjects when a subset of AMUSE components was retained to reconstruct the MEGs. Specificity was defined as the percentage of control subjects correctly detected, and sensitivity represented the rate of $\mathrm{AD}$ patients properly identified. Accuracy denotes the total fraction of subjects well recognized. In addition, (receiver-operating characteristic) ROC curves were plotted 
to visually assess the separation between groups in each case, and the corresponding area under the ROC curve (AROC) values were computed.

\section{RESULTS}

We applied the AMUSE algorithm to 20-seconds MEG epochs from $18 \mathrm{AD}$ patients and 18 controls. AMUSE ordered the components by decreasing linear predictability [5]. Thus, it was straightforward to compare AMUSE components between both subject groups. Every component was characterized by its $f_{\text {mean }}, S p E n, L Z C$, and $A p E n$ values.

A Student's $t$-test was carried out to statistically assess the significance of the differences between groups for the four variables. The $t$-test was applied separately to the values of each feature at every AMUSE component. The significance level was set at 0.01 . Table I shows the largest continuous range of AMUSE components that provided significant differences between both groups for each feature. As it can be seen, differences were significant for all variables at the AMUSE components ranked from 5 to 32. Hence, we chose this subset of 28 AMUSE components to partially reconstruct the MEGs. Then, these signals $\mathbf{x}_{p}(t)$ were used to classify the subjects. This case was denoted by BSS- $\{5,32\}$. For comparison, we also classified the $\mathrm{AD}$ patients and control subjects using the MEG recordings without any kind of BSS and component selection procedure.

For each of the two cases - without BSS and BSS- $\{5,32\}$ -, an average value of $f_{\text {mean }}, S p E n, L Z C$, and ApEn was computed per MEG channel and subject. Thus, for each feature, we obtained 148 values per subject. In order to reduce the problem dimensionality, we averaged the 148 values of each variable for every subject and case. Hence, the classification analysis was performed using one mean value of $f_{\text {mean }}, S p E n, L Z C$, and $A p E n$ per subject and case.

A LDA with a leave-one-out cross-validation procedure was applied to the four variables to model and classify the subjects for each case. Sensitivity, specificity, and accuracy values are shown in Table II for both cases (no BSS and BSS- $\{5,32\})$. The case BSS- $\{5,32\}$ improved the accuracy from $72.2 \%$ to $80.6 \%$. Moreover, the model selected by LDA when no BSS was used only contained $f_{\text {mean }}$, whereas in the case BSS- $\{5,32\}$ the data were modeled by both $L Z C$ and ApEn. In addition, Fig. 1 illustrates the ROC curves for each variable without BSS and with BSS-\{5,32\}. The AROC values are also shown in Fig. 1 for each case.

\section{DISCUSSION AND CONCLUSIONS}

In this study, AMUSE [17] was used to decompose MEG epochs of $18 \mathrm{AD}$ patients and 18 control subjects. Every component was described by its $f_{\text {mean }}$ [11], [12], $S p E n$ [12], [18], LZC [13], [19], and ApEn [14], [21] values. Student's $t$ test was applied to decide which components offered the most significant differences between subject groups. The results showed that 28 components, from 5 to 32, provided significant differences $(p<0.01)$ for all variables. Thus, the classification achieved with the partially reconstructed
TABLE I

SUBSETS OF AMUSE COMPONENTS WHICH PROVIDED STUDENT'S TTEST $P$-VALUES LOWER THAN 0.01

\begin{tabular}{lccccc}
\hline \hline Variable & $f_{\text {mean }}$ & SpEn & LZC & ApEn & $\begin{array}{c}\text { Common } \\
\text { range }\end{array}$ \\
\hline Range & 5 to 120 & 4 to 66 & 2 to 54 & 2 to 32 & 5 to 32 \\
\hline \hline
\end{tabular}

TABLE II

RESULTS FROM LDA WITH A LEAVE-ONE-OUT CROSS-VALIDATION PROCESS FOR CLASSIFICATION OF AD PATIENTS AND CONTROL SUBJECTS

\begin{tabular}{lccc}
\hline \hline & \multicolumn{3}{c}{ SUBJECTS } \\
& Sensitivity & Specificity & Accuracy \\
\hline Without BSS & $72.2 \%$ & $72.2 \%$ & $72.2 \%$ \\
With BSS- $\{5,32\}$ & $72.2 \%$ & $88.9 \%$ & $80.6 \%$ \\
\hline \hline
\end{tabular}

MEGs - BSS- $\{5,32\}$ - was compared to that obtained without BSS-preprocessing. The accuracy improved an $8.4 \%$ (from $72.2 \%$ to $80.6 \%$ ). Moreover, it should be noted that the component selection and the classification were carried out with different techniques. Whereas the first one was based on the Student's $t$-test, the classification was assessed with a LDA with a leave-one-out cross-validation procedure. Nevertheless, it is important to note that other basic BSS algorithms [1], such as principal component analysis, and other feature selection techniques might be also applied to improve the classification of subject groups.

In contrast to other applications of BSS to EEG or MEG data, we did not aim to isolate specific physiological activity [2]-[4]. Instead, we wanted to enhance the differences between $\mathrm{AD}$ patients and control subjects' MEG recordings by retaining only the most relevant components [5].

The ROC curves depicted in Fig. 1 show clear improvements in the differentiation between $\mathrm{AD}$ patients and control subjects for three variables ( $S p E n, L Z C$, and $A p E n)$. Nevertheless, the AROC value did not increase appreciably for $f_{\text {mean }}$. This could be due to the fact that the component selection was based on all variables. Thus, it is likely that this process did not optimize the subject differentiation based on every single variable.

To sum up, our results suggest that a BSS and component selection procedure may be useful to improve the classification between AD patients and control subjects' MEGs. This agrees with studies from other authors [5]. However, our results must be confirmed analyzing a larger database with AMUSE and other BSS algorithms.

\section{REFERENCES}

[1] A. Cichocki and S. Amari, Adaptive blind signal and image processing: learning algorithms and applications. Cichester, West Sussex, England: John Wiley and Sons, 2003.

[2] C. J. James and C. W. Hesse, "Independent component analysis for biomedical signals," Physiol. Meas., vol. 26, pp. R15-R39, 2005.

[3] J. Escudero, R. Hornero, D. Abásolo, A. Fernández, and M. LópezCoronado, "Artifact removal in magnetoencephalogram background activity with independent component analysis," IEEE Trans. Biomed. Eng., to be published. Available: doi: 10.1109/TBME.2007.894968.

[4] K. H. Ting, P. C. W. Fung, C. Q. Chang, and F. H. Y. Chan, "Automatic correction of artefact form single-trial event-related potentials by blind source separation using second order statistics only," Med. Eng. Phys., vol. 28, pp. 780-794, 2006. 


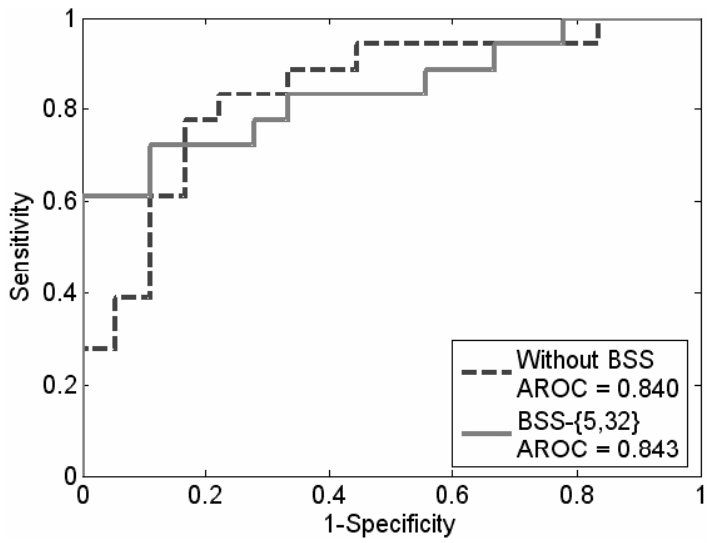

(a)

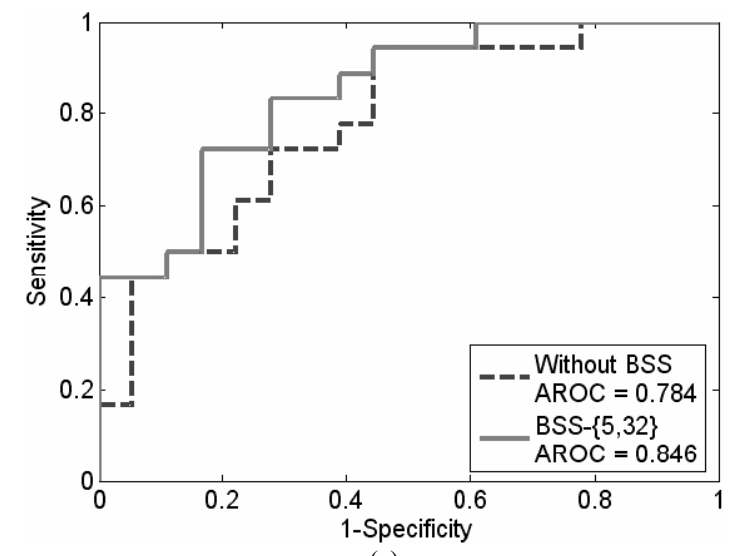

(c)

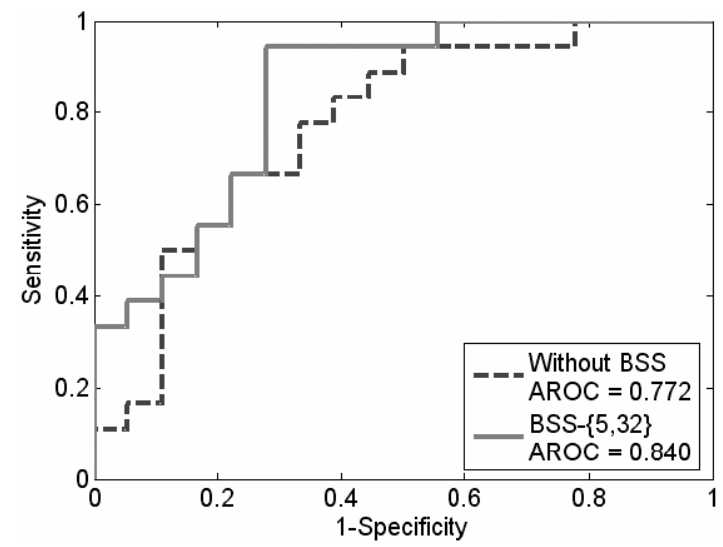

(b)

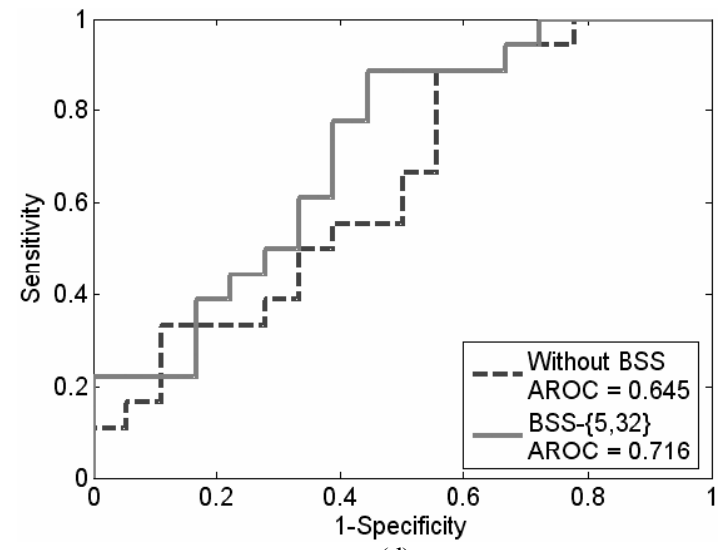

(d)

Fig. 1. ROC curves illustrating the ability of the features included in this study to distinguish AD patients from control subjects. Comparison with (grey full curve) and without (black dashed curve) the BSS and component selection procedure is plotted. For each case, the AROC value is also shown. (a) $f_{\text {mean. }}$ (b) SpEn. (c) LZC. (d) ApEn.

[5] A. Cichocki, S. L. Shishkin, T. Musha, Z. Leonowicz, T. Asada, and T. Karachi, "EEG filtering based on blind source separation (BSS) for early detection of Alzheimer's disease," Clin. Neurophysiol., vol. 116, pp. 729-737, 2005.

[6] S. H. Jin, J. Jeong, D. G. Jeong, D. J. Kim, S. Y. Kim, "Nonlinear dynamics of the EEG separated by independent component analysis after sound and light stimulation," Biol. Cybern., vol. 86, pp. 395-401, 2002.

[7] T. D. Bird, "Alzheimer's disease and other primary dementias," in Harrison's Principles of Internal Medicine, E. Braunwald, A. S. Fauci, D. L. Kasper, S. L. Hauser, D. L. Longo, and J. L. Jameson, Eds. New York: McGraw-Hill, 2001, pp. 2391-2399.

[8] D. J. Selkoe, “Alzheimer's disease: genes, proteins, and therapy," Physiol. Rev., vol. 81, pp. 741-766, 2001.

[9] J. Jeong, "EEG dynamics in patients with Alzheimer's disease," Clin. Neurophysiol., vol. 115, pp. 1490-1505, 2004.

[10] R. Hari, "Magnetoencephalography in clinical neurophysiological assessment of human cortical functions," in Electroencephalography: Basic Principles, Clinical Applications, and Related Fields, 5th ed., E. Niedermeyer, and F. Lopes da Silva, Ed. Philadelphia: Lippincott Williams \& Wilkins, 2005, pp. 1165-1197.

[11] M. Signorino, E. Pucci, N. Belardinelli, G. Nolfe, and F. Angeleri, "EEG spectral analysis in vascular and Alzheimer dementia," Electroencephalogr. Clin. Neurophysiol., vol. 94, pp. 313-325, 1995.

[12] J. Poza, R. Hornero, D. Abásolo, A. Fernández, and M. García, "Extraction of spectral based measures from MEG background oscillations in Alzheimer's disease," Med. Eng. Phys., to be published. Available: doi:10.1016/j.medengphy.2006.11.006.

[13] C. Gómez, R. Hornero, D. Abásolo, A. Fernández, and M. López, "Complexity analysis of the magnetoencephalogram background activity in Alzheimer's disease patients," Med. Eng. Phys., vol. 28, pp. 851-859, 2006.
[14] D. Abásolo, R. Hornero, P. Espino, J. Poza, C. I. Sánchez, and R. de la Rosa, "Analysis of regularity in the EEG background activity of Alzheimer's disease patients with approximate entropy," Clin. Neurophysiol., vol. 116, pp. 1826-1834, 2005.

[15] G. McKhann, D. Drachman, M. Folstein, R. Katzman, D. Price, and E. M. Stadlan, “Clinical diagnosis of Alzheimer's disease: report of the NINCDS-ADRDA Work Group under the auspices of Department of Health and Human Services Task Force on Alzheimer's disease," Neurology, vol. 34, pp. 939-944, 1984.

[16] M. F. Folstein, S. E. Folstein, and P. R. McHugh, "Mini-mental state. A practical method for grading the cognitive state of patients for the clinician," J. Psychiatr. Res., vol. 12, pp. 189-198, 1975.

[17] L. Tong, R. Liu, V. C. Soon, and Y.-F. Huang, "Indeterminacy and identifiability of blind identification," IEEE Trans. Circuits Syst., vol. 38, pp. 499-509, 1991.

[18] T. Inouye, K. Shinosaki, H. Sakamoto, S. Toi, S. Ukai, A. Iyama, Y. Katsuda, and M. Hiramo, "Quantification of EEG irregularity by use of the entropy of the power spectrum," Electroencephalogr. Clin. Neurophysiol., vol. 79, pp. 204-210, 1991.

[19] A. Lempel and J. Ziv, "On the complexity of finite sequences," IEEE Trans. Inf. Theory, vol. IT-22, pp. 75-81, 1976.

[20] M. Aboy, R. Hornero, D. Abásolo, and D. Álvarez, "Interpretation of the Lempel-Ziv complexity measure in the context of biomedical signal analysis," IEEE Trans. Biomed. Eng., vol. 53, pp. 2282-2288, 2006.

[21] S. M. Pincus, "Approximate entropy as a measure of system complexity," Proc. Natl. Acad. Sci. USA, vol. 88, pp. 2297-2301, 1991

[22] R. Hornero, M. Aboy, D. Abásolo, J. McNames, and B. Goldstein, "Interpretation of approximate entropy: analysis of intracranial pressure approximate entropy during acute intracranial hypertension," IEEE Trans. Biomed. Eng., vol. 52, pp. 1671-1680, 2005. 